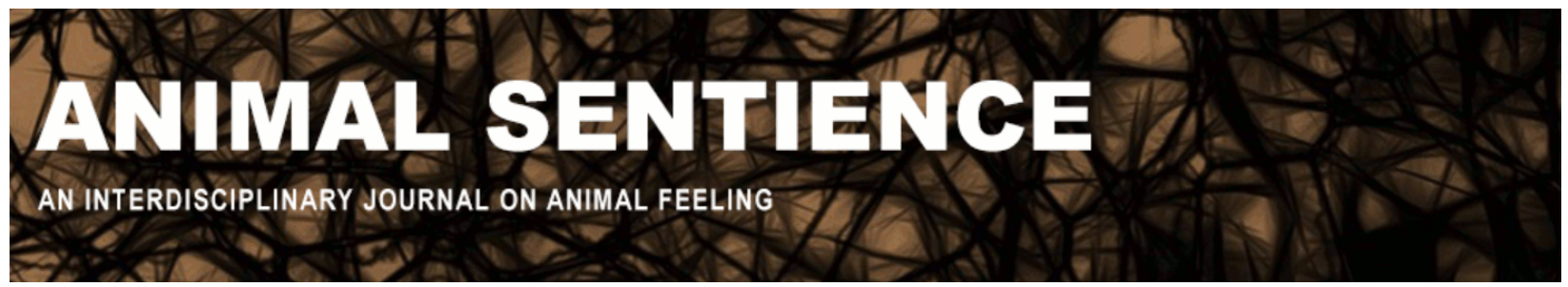

Gerlai, Robert (2017) Learning, memory, cognition, and the question of sentience in fish. Animal Sentience 13(8)

DOI: $10.51291 / 2377-7478.1242$

Date of submission: 2017-11-02

Date of acceptance: 2017-11-06

(c) $\underset{\mathrm{EY}}{\mathrm{C}}$

This article has appeared in the journal Animal

Sentience, a peer-reviewed journal on animal

cognition and feeling. It has been made open access,

free for all, by WellBeing International and deposited

in the WBI Studies Repository. For more information,

please contact

wbisr-info@wellbeingintl.org.

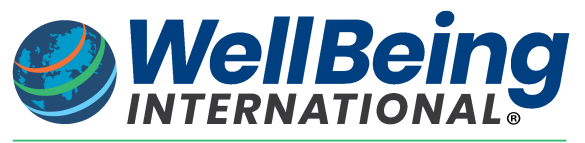

SOLUTIONS FOR PEOPLE, ANIMALS AND ENVIRONMENT 


\title{
Learning, memory, cognition, and the question of sentience in fish
}

\author{
Commentary on Woodruff on Fish Feel
}

\author{
Robert Gerlai \\ Department of Psychology \\ University of Toronto Mississauga
}

\begin{abstract}
Evolutionarily conserved features have been demonstrated at many levels of biological organization across a variety of species. Evolutionary conservation may apply to complex behavioral phenomena too. It is thus not inconceivable that a form of sentience does exist even in the lowest order vertebrate taxon, the teleosts. How similar it is to human sentience in its level of complexity or in its multidimensional features is a difficult question, especially from an experimental standpoint, given that even the definition of human sentience is debated. Woodruff attempts a Turing-like test of fish sentience, and lists numerous neuroanatomic, neurophysiological and behavioral similarities between fish and humans. In this commentary, I add to these similarities by discussing empirical findings showing complex forms of mental representation in fish. At the same time, I note that without a more thorough understanding of human sentience and more data on similarities as well as differences between fish and mammals, the final conclusion may have to wait.
\end{abstract}

Robert Gerlai, Professor of Psychology, University of Toronto, does research on neurobiological and genetic mechanisms of behavior in fish and rodents. He is Fellow and past president of the International Behavioral Neuroscience Society. He received the Distinguished Scientist Award from the International Behavioral and Neural Genetics Society in 2013, and the University of Toronto Mississauga Excellence in Research Award in 2015. sites.utoronto.ca/GerlaiLab/

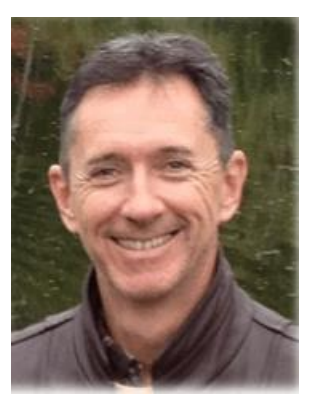

\section{Evolutionary conservation at many levels of biology: Does it extend to sentience?}

Evolutionary biology has taught us that all species are related to each other. The question is only the degree of relatedness. We also know that evolution is a process whereby random genetic changes leading to alterations in previously existing phenotypes are tested in terms of how they increase or decrease the ability to reproduce under current environmental conditions. In other words, evolution is not a designer that creates things from scratch. Biologists find evolutionary homologies across a broad range of features and across a large number of species from fish to human. Steps of embryonic development, fundamental anatomical layout of brains, neurotransmitter systems and their receptors, molecular mechanisms of neuronal plasticity, and amino acid sequences of proteins all exhibit homologies, which are signs of common ancestry.

Why should sentience be different? I would argue, therefore, that the question is not whether fish are sentient, but rather to what degree and in what flavor? In other words, where 
do we draw the line? Or more provocatively, is there a line to draw at all? Clearly, human sentience is a species-typical feature of Homo sapiens. Fish do not have it. But does this prove that we are the only sentient creature walking (flying, swimming, crawling) on this planet? Woodruff's (2017) target article makes a genuine effort to show us that it does not!

\section{Where do we draw the line? A Turing test for fish.}

According to Woodruff, fish do exhibit signs of sentience, a conclusion that depends upon how we define the phenomenon and associated underlying mechanisms. And there lies the biggest question: the definition of the phenomenon. We all think we know what it is to be aware, or to be sentient or conscious. But does science really know the answer to these questions? Woodruff attempts to answer by focusing on empirical, experimental definitions: mechanisms and behavioral responses, a Turing test (Turing, 1950) of sentience for fish. He shows us that fish do indeed possess features at the levels of anatomy, neurophysiology and behavior compatible with the assumption of the presence of sentience. The similarities he cites across human and fish features abound. But so do the differences (Bayés et al., 2017)! Which should we emphasize? And which represent proof or disproof?

My own impression is that these questions are still to be discussed, and the answers will continue to be refined and re-discussed again and again. But let me add another angle to this discussion, leading back to the question of how we define sentience. To me, it appears this phenomenon has to do with modeling the external world. Mental representations of the outside allow the organism to remember the past, understand the present, and forecast the future, a phenomenon that in humans, and in some other mammals as well as some bird species, scientists call the ability to do "mental time travel" (Clayton, 2015). Mental representations imply that there is an actor, the self, that experiences, and plays a role in, the representation. Sentience may be an epiphenomenon, an emergent property of increasingly sophisticated mental representations afforded by complex brains. Setting aside questions about specific anatomy, connectome, electrophysiology and molecular mechanisms, do we know whether fish brains are complex enough for mental representations to form? From the results of behavioral studies, the answer to this question is: yes, we already do.

\section{Complex mental representations: What do fish learn and remember?}

I will give only two examples, from my own research. The ability to make numerical or quantity estimations is a human feature but it is also widespread in the animal kingdom (Feigenson et al., 2004). A variety of fish species have been shown to be able to tell more from less, and, surprisingly, the way they do it also resembles how human children do it (Gómez-Laplaza \& Gerlai, 2016). Most recently, we have shown that a fish species, angelfish (Pterophyllum scalare), is not only capable of making the distinction between more and less when the item sets to be compared are present at the same time, but also when the items are not shown, i.e., when the fish have to remember where the items used to be (Gómez-Laplaza \& Gerlai, 2016). In other words, we now have clear evidence of memory, i.e., mental representation of more vs. less in a fish species. Does this mean that fish "understand" the abstract meaning of more vs. less? are they "aware" of their 
choices? No, it does not. But it does show a level of sophistication in their mental representation abilities that in the past was thought to be uniquely human.

The other example of complex mental representation in fish comes from our studies on spatial learning in zebrafish. As also discussed by Woodruff, the hallmark of episodic or declarative memory is its relational aspect (Eichenbaum, 1992). The ability to organize and relate what seem to be loosely related bits and pieces of information is a fundamental requirement for us to be able to remember where, what and when things happened to us, a function supported by the hippocampal formation. Thus, tests of relational memory have been used to analyze "declarative episodic" memory in non-human animals too (Eichenbaum, 1992), species that cannot talk. A frequently employed relational type of hippocampal memory task is the spatial memory test in which mammals (mostly rodents) learn the dynamic representation of visual cues outside the maze.

But how do we know what they actually learn? This is a complicated question that has been explored experimentally. A rodent with a disrupted hippocampus can pick out a single cue from the background, turning the spatial task into an elemental task that it can seemingly perform well (Gerlai, 1998; Phillips \& LeDoux, 1994). However, if a salient associative cue is experimentally provided in addition to the spatial cues, hippocampally lesioned animals cannot pick out a secondary cue, and thus will "recognize" the location of the reinforcer only when the associative cue is presented. Hippocampally intact mammals, on the other hand, can learn both at the same time: the dynamic spatial map, and the salient cue.

Thus, when faced with the spatial task, hippocampally intact rodents can identify the location of the reinforcer even in the absence of the salient associative cue (Gerlai, 1998; Phillips \& LeDoux, 1994). Surprisingly, so can zebrafish (Karnik \& Gerlai, 2014). In other words, zebrafish acquire spatial information the same way rodents with an intact hippocampus do, despite the fact that zebrafish lack the hippocampal structure specific to mammalian brains. The ability to acquire a complex spatial map suggests sophisticated mental representation in fish. Does it mean fish are sentient? No, it does not. But this second Turing test of mental representation previously thought to be a sole property of complex mammalian brains has now also been passed with flying colors by fish.

In sum, Woodruff's arguments about the many similarities between fish and humans are eyeopening. But to truly understand what it feels like to be a fish, we may need to appreciate both the similarities and the differences between us and them. We may even need a better understanding of what it really feels like to be human.

\section{References}

Bayés, À., Collins, M.O., Reig-Viader, R., Gou, G., Goulding, D., Izquierdo, A., Choudhary, J.S., Emes, R.D., \& Grant, S.G. (2017). Evolution of complexity in the zebrafish synapse proteome. Nat Commun 8:14613. doi: 10.1038/ncomms14613

Clayton, N. S. (2015) Ways of thinking: From crows to children and back again. Q J Exp Psychol 68(2):209-241. 
Eichenbaum, H. (1992). The hippocampal system and declarative memory in animals. J Cogn Neurosci 4(3):217-231.

Feigenson, L., Dehaene, S., \& Spelke, E.S. (2004). Core systems of number. Trends Cogn Sci 8:307-314.

Gerlai, R. (1998). Contextual learning and cue association in fear conditioning in mice: A strain comparison and a lesion study. Behav Brain Res 95:191-203.

Gómez-Laplaza, L.M., \& Gerlai, R. (2016). Discrimination of large quantities: Weber's law and short-term memory in angelfish (Pterophyllum scalare) Animal Behaviour 112:29-37.

Karnik, I., \& Gerlai, R. (2012). Can zebrafish learn spatial tasks? An empirical analysis of place and single CS-US associative learning. Behav Brain Res 233:415-421.

Phillips, R.G., \& LeDoux, J.E. (1994). Lesions of the dorsal hippocampal formation interfere with background but not foreground contextual fear conditioning. Learning \& Memory 1:34-44.

Turing, A. (1950). Computing machinery and intelligence. Mind, LIX 236:433-460.

Woodruff, M. L. (2017). Consciousness in teleosts: There is something it feels like to be a fish. Animal Sentience 13(1). 


\section{ANIMAL CONSCIOUSNESS}

On November 17-18, 2017, the NYU Center for Mind, Brain and Consciousness, the NYU Center for Bioethics, and NYU Animal Studies will host a conference on Animal Consciousness.

This conference will bring together philosophers and scientists to discuss questions such as: Are invertebrates conscious? Do fish feel pain? Are nonhuman mammals self-conscious? How did consciousness evolve? How does research on animal consciousness affect the ethical treatment of animals? What is the impact of issues about animal consciousness on theories of consciousness and vice versa? What are the best methods for assessing consciousness in nonhuman animals?

\section{Speakers and panelists include:}

Colin Allen (University of Pittsburgh, Department of History \& Philosophy of Science), Andrew Barron (Macquarie, Cognitive Neuroethology),

Victoria Braithwaite (Penn State, Biology), Peter Carruthers (Maryland,

Philosophy), Marian Dawkins (Oxford, Zoology), Dan Dennett (Tufts,

Philosophy), David Edelman (San Diego, Neuroscience),

Todd Feinberg (Mt. Sinai, Neurology), Peter Godfey-Smith (Sydney,

Philosophy), Lori Gruen (Wesleyan, Philosophy), Brian Hare (Duke, Evolutionary

Anthropology), Stevan Harnad (Montreal, Cognitive Science), Eva Jablonka (Tel

Aviv, Cohn Institute), Björn Merker (Neuroscience), Diana Reiss (Hunter,

Psychology), Peter Singer (Princeton, Philosophy), Michael Tye (Texas, Philosophy)

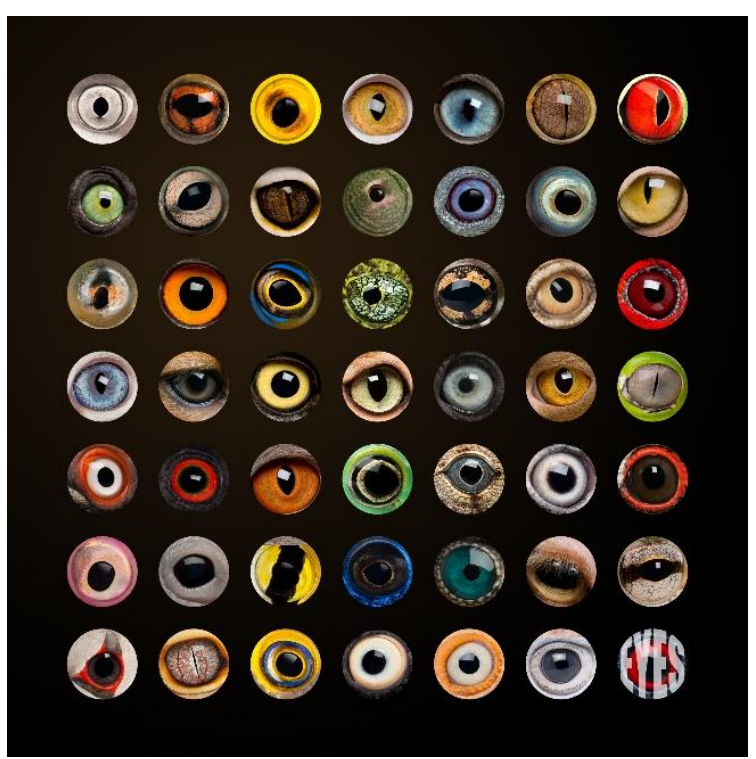

Organizers: Ned Block, David Chalmers, Dale Jamieson, S. Matthew Liao.

The conference will run from 9am on Friday November 17 to $6 \mathrm{pm}$ on Saturday November 18 at the NYU Cantor Film Center (36 E 8th St).

Friday sessions will include "Invertebrates and the evolution of consciousness", "Do fish feel pain?", and "Animal consciousness and ethics".

Saturday sessions will include "Animal self-consciousness", "Animal consciousness and theories of consciousness", and a panel discussion.

A detailed schedule will be circulated closer to the conference date.

Registration is free but required.

\section{Register here.}

\section{See also the conference website}

